\title{
Defect-governed double-step activation and directed flame fronts
}

\author{
Egor V. Yakovlev, ${ }^{1}$ Nikita P. Kryuchkov, ${ }^{1}$ Pavel V. Ovcharov, ${ }^{1}$ \\ Killian Pitiot, ${ }^{1}$ Andrei V. Sapelkin, ${ }^{2}$ and Stanislav O. Yurchenko, ${ }^{1}+$ \\ ${ }^{1}$ Bauman Moscow State Technical University, 2nd Baumanskaya street 5, 105005 Moscow, Russia \\ ${ }^{2}$ Queen Mary University of London, Department of Physics, E14NS London, England
}

(Dated: September 17, 2019)

\begin{abstract}
Defects play a crucial role in physics of solids, affecting their mechanical, electromagnetic, and chemical properties. However, influence of thermal defects on wave propagation in exothermic reactions (flame fronts) still remains poorly understood at molecular level. Here, we show that thermal behavior of the defects exhibits essential features of double-step exothermic reactions with pre-equilibrium. We use experiments with monolayer complex (dusty) plasma and find that it can show a double-step activation thermal behavior, similar to chemically-reactive media. Furthermore, for the first time we demonstrate capabilities to control flame fronts using defects and the different dynamic regimes of the thermal defects in complex (dusty) plasmas, from non-activated one to being sound- and self-activated (like in active soft matter). The results suggest that a range of challenging phenomena at the forefront of modern science (e.g. defect activation, flame front dynamics, reaction waves etc.) can now be experimentally interrogated on a microscopic scale.
\end{abstract}

\section{INTRODUCTION}

Defects play essential role in physics of crystals, owing to their effect on mechanical, thermal, electric, magnetic, optical, and chemical properties. Over the last few decades, their importance has been growing and extended to condensed matter, physical chemistry, chemical physics, electro- and mechanochemistry, soft matter, materials science, and fuel technologies [1 3]. However, today thermal activation of defects in chemicallyreactive solids remains poorly understood on the microscopic scale, since in typical materials molecules or atoms cannot, in general, be observed directly, while the corresponding numerical simulations from the first principles are excessively time-consuming. Besides, verifying such calculations on the atomic/molecular scale experimentally is still a major challenge. Hence, there is a clear demand for a flexible model system capable of delivering particle-resolved information on defect behaviour in a variety of condensed matter scenarios and for which the corresponding theoretical model can be made readily available. We believe that one such system is complex (dusty) plasma.

It is well-known, that physical insights into mechanisms governing generic phenomena at the level of individual particles in fluids and solids can be obtained with complex (dusty) plasmas - plasma state of soft matter consisted of strongly-interacting (charged) microparticles immersed in a weakly-ionized gas 4 6]. These systems can exhibit molecular-like behavior and have already provided crucial insights into such generic phenomena as melting [7-11], crystallization [12, 13], heat transport [14, 15, dynamics of dislocations [16, 17, glassy state [18, 19], and spinodal decomposition [20, 22]. Recently, it has been shown that complex (dusty) plasmas can serve

\footnotetext{
* st.yurchenko@mail.ru
}

as an open system with tunable thermal activation behavior controlled experimentally. This enables investigation of phenomena intrinsic for chemically-reactive media such as propagation of flame fronts [23, 24, thermal explosion [25, and generation of acoustic pulsations due to thermoacoustic instability [26. Due to the visualization of individual microparticles, complex plasmas present a unique opportunity for particle-resolved analysis of complex phenomena, which are analogous to ones in chemically-reactive media including behavior of thermal defects in solids.

In this paper, we introduce for the first time defects into an artificially constructed $2 \mathrm{D}$ crystal. We then study defect activation and their role in propagation of flame fronts in solids. We observed a phenomenon when acoustic pulsations that originate from thermoacoustic instability during propagation of flame fronts, induce thermal explosion of the defects. We show that defects reduce the activation temperature threshold, change kinetics of energy release, and affect the shape of flame front, while the corresponding thermal behavior reveals features we attribute to exothermic double-step chemical reactions (activation with pre-equilibrium). Using our experimental system, we demonstrate that flame fronts can be directed and even halted by defects. Moreover, molecular dynamics (MD) simulations provide evidence that the thermal defects in complex (dusty) plasmas exhibit a range of dynamic regimes, from non-activated one to being soundand self-activated. We believe that results of this work open exciting prospects for a variety of current problems in energy materials, physical chemistry, chemical physics, and soft matter.

\section{SOUND-INDUCED ACTIVATION OF THERMAL DEFECTS}

We performed a number of dedicated experiments (see details of the experimental set up in Appendix A with 

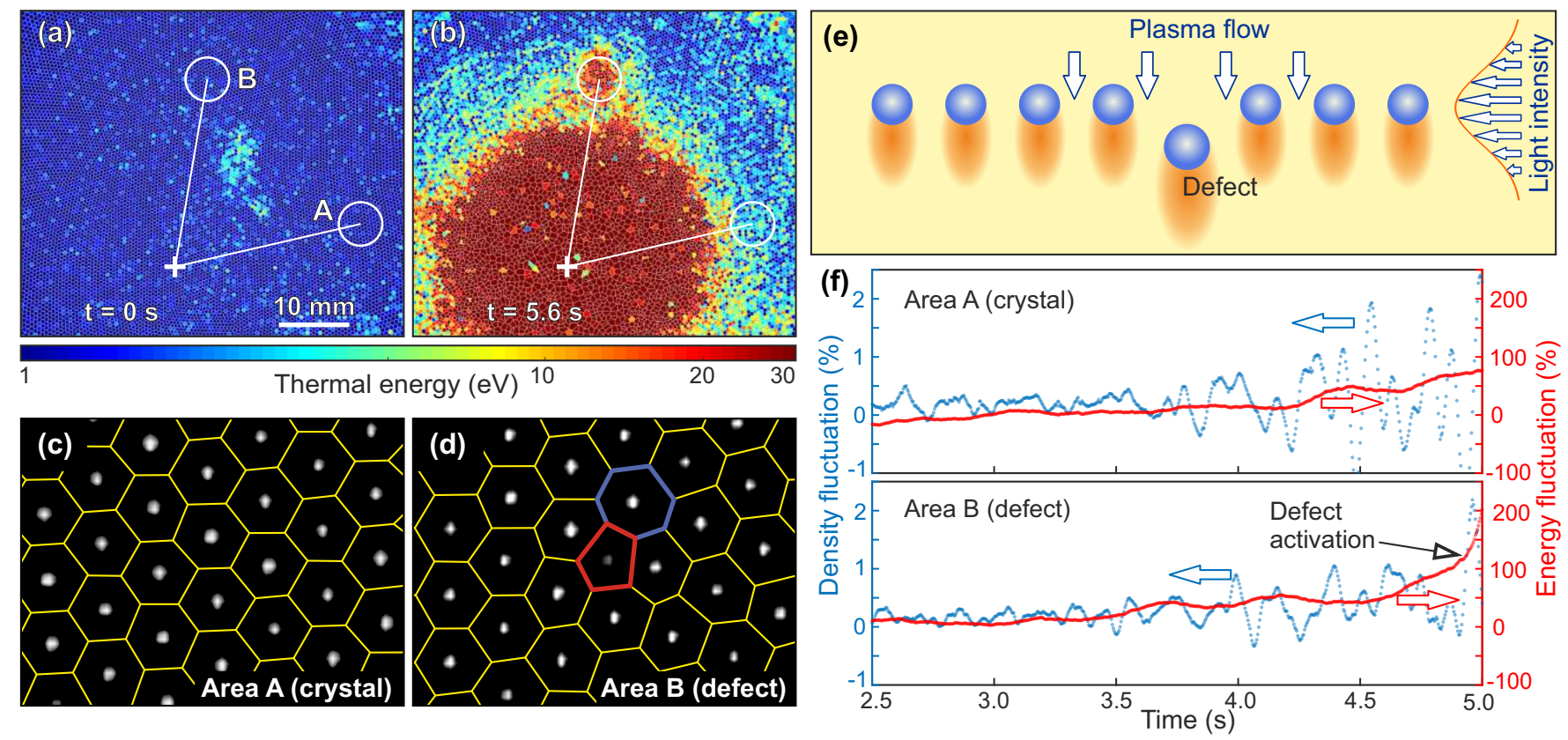

FIG. 1. Thermal defect activation during propagation of "flame front" in complex (dusty) plasma crystal: (a, b) Evolution of thermal energy of particles. (c, d) Snapshots of the areas A and B marked in (a), corresponding to the areas of crystal and a havier particle (thermal defect), at the moment $t=-1 \mathrm{~s}$. Yellow solid lines are color-coded Voronoi cells, corresponding to six (yellow), five (red), and seven (blue) neighbors. The darker particle in the five-fold cell in (d) is the thermal defect. (e) Illustration why heavier particle (thermal defect) is less lighted by the laser sheet in the layer. (f) Evolution of thermal energy and relative density fluctuations in the areas A and B marked in (a). See Movie S1 [27.

the results of our investigations into experimentallyobserved thermal activation of a defect during the propagation of "flame fronts" (fronts of nonequilibrium melting of the plasma crystal supported by thermal activation of the fluid plasma behind the front [23]) in complex (dusty) plasma crystal presented in Fig. 1.

Figures 1(a) and (b) show snapshots of thermal energy distribution at $t=0$ and $5.6 \mathrm{~s}$ (see Supplemental Movie S1 [27]). Here, we performed Voronoi decomposition for the studied system and colored each cell in accordance with its thermal energy. Figures 1.(c) and (d) combine experimental images with boundaries of Voronoi cells for area A (crystal) and B (defect) at $t=0 \mathrm{~s}$. The heavier particle appears as a darker particle in Fig. 1(d) since its equilibrium position is below the plane of plasma crystal (as illustrated in Fig. 1(e)). Such arrangement plays a crucial role in thermal behavior of defects modelled by such heavier particles.

In our experiments with monolayer complex (dusty) plasmas, the interactions between particles are mediated by plasma flows owing to formation of plasma wakes downstream of each particle [4, 6, as shown schematically by the orange clouds in Fig. 1(e). The wakes play the role of a third body and violate reciprocity of (effective) action and reaction forces acting between particles 28 31. As a consequence, dynamics of the system is determined by interplay between energy release (due to nonreciprocity of interactions) and dissipation (due to
Epstein damping) [6, 30, 32.

It has been shown that, under weak damping conditions, plasma wakes can induce mode-coupling instability (MCI) in plasma crystals [24, 33, 34] and fluids [35], when the energy of plasma flow is transferred to the microparticle subsystem if the in- and out-of-plane collective modes intersect. The crystalline MCI was used in these experiments to initiate the melting of the crystal: once the crystal is intensively heated due to the crystalline MCI ("ignited") in the central (densest) part, radially-expanding front of nonequilibrium melting (flame front, Fig. 1(b)) was formed, similarly to Refs. [10, 23, 25, 26]. Simultaneously, intensive acoustic pulsations were generated from the melted region due to thermoacoustic instability [26], leading to intensive density fluctuations which can be seen in Fig. 1(f). One can see strong density fluctuation in both areas A (no defect) and B (defect) after around $t=4.0 \mathrm{~s}$, however, only at the defect location site $\mathrm{B}$ these fluctuations result in rapid energy increase at $t=4.9 \mathrm{~s}$ in Fig. 1(f). Thus, density fluctuations around the defect site lead to temperature increase above its critical (threshold) value, trigger "thermal explosion" (a local sharp rise in thermal energy of the particles) [25], and curve the flame front, as can be seen in Fig. 1(b) and in Movie S1 27]. This is a consequence of the non-reciprocal interparticle interaction mediated by plasma wakes (see Fig. (8) that, in the presence of an acoustic wave, results in an efficient and localized energy transfer to the de- 


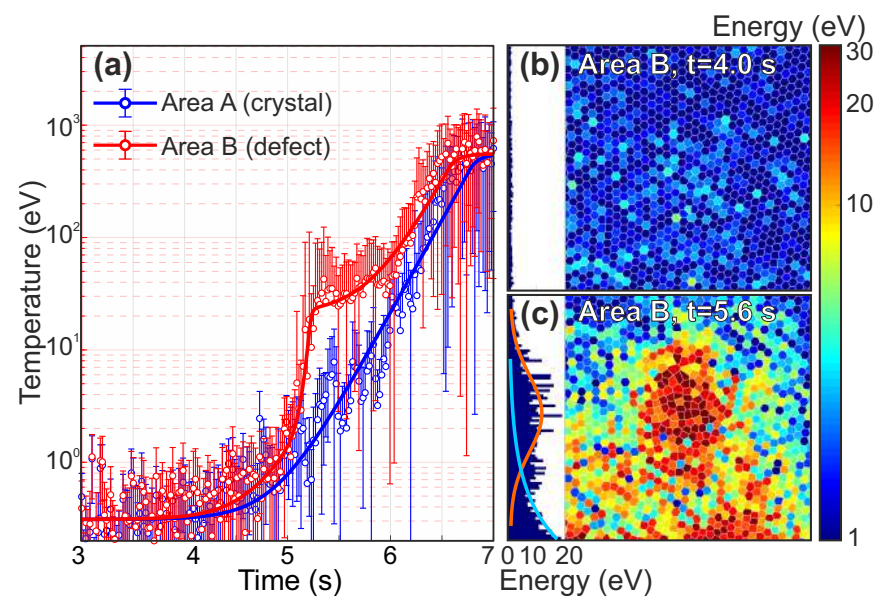

FIG. 2. Experimentally-observed double-step thermal activation: (a) Evolution of thermal energy (temperature) in crystals and in vicinity of a defect. Symbols and bars are experimental results, solid lines are theoretical fits (4). (b, c) Area B (thermal defect) before and after its activation. The blue and orange lines in (b) are exponential and gaussian thermal profiles, respectively. See Movie S1 [27].

fect. The defect activation is associated with the process of the plasma crystal melting near the heavier particle, when its energy reaches the threshold value (the activation energy).

The experimental results clearly demonstrate a complex activation behavior and a cascade of the thermal instabilities in our system. During the first stage, the MCI develops in the crystal [33 35, that results in formation of melted region. This is followed by MCI in the complex plasma fluid [35], that leads to formation of steady flame front [10, 23]. This flame front is accompanied by intensive sound generation (pulsations) from the melted region owing to thermoacoustic instability [26]. Finally, the pulsations trigger thermal explosion [25] of the defect before the arrival of the flame front.

To test that the observed behavior is indeed due to the "reactivity" of the media provided by nonreciprocal interactions between particles (due to mediation of the interaction by the plasma flow), we performed MD simulations under conditions corresponding to our experiment. This has been done in the manner previously reported in Refs. 23, 26] (see details in Appendix B). The results are presented in Fig. 3 and show a remarkable similarity with Fig. 2 (see Movie S2 27]). We found that all experimentally observed features of thermal activation of the defects are reproduced in MD simulation very well supporting our interpretation of the experimental results.

\section{REACTIONS WITH PRE-EQUILIBRIUM}

Further analysis revealed that thermal evolution of the defect exhibits double-step behavior, similar to chemical reactions with pre-equilibrium (here, we mean the reac-

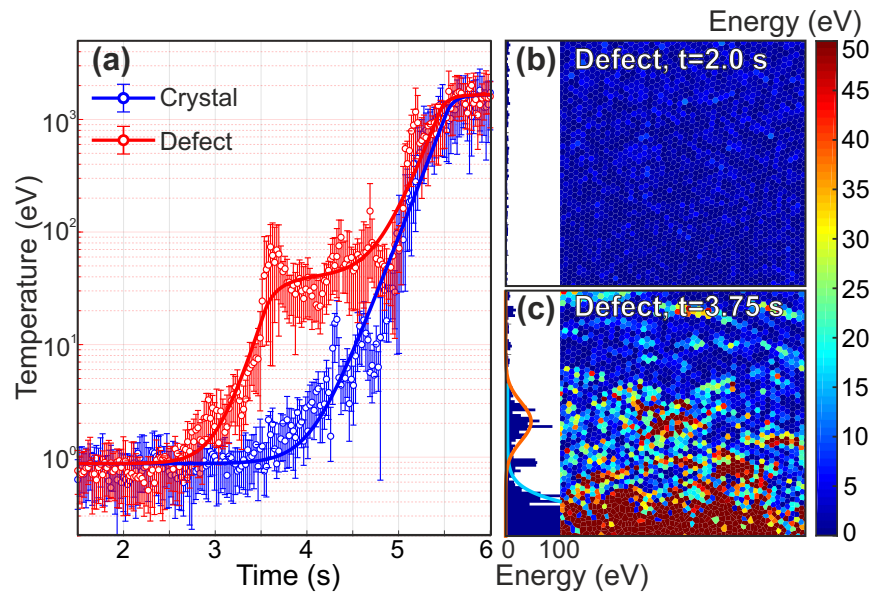

FIG. 3. MD results for double-step thermal activation of the defect: Description is the same as in Fig. 2 See Movie S2 27.

tions with an intermediate long-living state) [36]. This double-step behaviour is particularly clear once we compare the time evolution of average temperatures (see Fig. 2(a)) in the areas A and B in Fig. 1(a). Symbols and bars in Fig. 2(a) correspond to the temperatures and their standard deviations calculated in the areas A (blue) and B (red), respectively. Lines are theoretical fits, obtained using one- and two-step chemical reaction models (exothermic reactions with pre-equilibrium [36]), respectively (see discussion below). Moreover, one should note that the defect becomes thermally-activated before the flame front reached the defect (at the distance to the main front is about 35 lattice constants or $\sim 15 \mathrm{~mm}$ ).

We found that the observed cascades of nonequilibrium phase transitions provide a physical analogy with stepwise exothermic reaction with two steps (activation with pre-equilibrium) 36. Indeed, consider the heat equation [37:

$$
\frac{\partial T}{\partial t}=\frac{Q(T)}{C n}+\chi \Delta T,
$$

where $T(r, t)$ is the spatial temperature distribution, $Q(T)$ is the heat rate of the medium, $C$ and $n$ are the isobaric heat capacity (per particle) and the number density, respectively; $\chi$ is the thermal diffusivity.

In reactive media, the heat source $Q(T)$ is determined by kinetics of the exothermic chemical reactions [36. In case of the double-step chemical reactions one can assume that $Q(T)$ is

$$
Q(T)=Q_{1}(T)+Q_{2}(T),
$$

where the two terms on the right correspond to the first and the second steps, which can be approximated as

$$
\begin{aligned}
\frac{Q_{1}(T)}{C n} & = \begin{cases}-\alpha_{1}\left(T-T_{0}\right), & T<T_{A 1} ; \\
q_{1}-\alpha_{1}\left(T-T_{0}\right), & T_{A 1}<T<T_{1},\end{cases} \\
\frac{Q_{2}(T)}{C n} & = \begin{cases}-\alpha_{2}\left(T-T_{1}\right), & T_{1}<T<T_{A 2} ; \\
q_{2}-\alpha_{2}\left(T-T_{1}\right), & T_{A 2}<T<T_{2},\end{cases}
\end{aligned}
$$


where $T_{A 1,2}$ and $q_{1,2}$ (indices describe the first and second step of the reaction) are the activation temperatures and the energy releases, parameters $\alpha_{1,2}$ are related to details of chemical kinetics, $T_{0}, T_{1}$, and $T_{2}$ are background and saturation temperatures at the fist and second steps, respectively.

Considering activations at the first and second steps separately (a posteriori analysis justifies this assumption), we obtain the following time-dependence of temperature (see details in Appendix C)

$$
\begin{aligned}
T(t) \simeq T_{0}+\left(T_{1}-\right. & \left.T_{0}\right) f\left(t-t_{1}, \lambda_{1}, \lambda_{2}\right)+ \\
& +\left(T_{2}-T_{1}\right) f\left(t-t_{2}, \lambda_{3}, \lambda_{4}\right),
\end{aligned}
$$

where $t_{1}$ and $t_{2}$ are moments of the first and second step activations, $\lambda_{1 \ldots 4}$ are the parameters related to the flame front velocity, energy releases, and saturation temperatures, and

$$
f\left(t, \lambda, \lambda^{\prime}\right)= \begin{cases}\frac{e^{\lambda t}}{1+\lambda / \lambda^{\prime}}, & t<0 \\ 1-\frac{\lambda}{\lambda^{\prime}} \frac{e^{-\lambda^{\prime} t}}{1+\lambda / \lambda^{\prime}}, & t>0\end{cases}
$$

In the particular case of $T_{1}=T_{0}$, solution (4) coincides with the profile of flame fronts reported in Refs. 23, 38. for one-step reaction.

The state energy vs reaction coordinate for reactions with simple (one-step) activation and for more complicated case of activation with pre-equilibrium (stepwise exothermic reaction with two steps) are schematically illustrated in Fig. 4. In reactive materials, mechanisms of stepwise reactions can be related to generation of intermediates, to partial change in chemical bounds, and to complex chemical reactions [36]. A simple case is characterized by the activation energy $E_{\mathrm{A}}$ and energy release $E$, as shown by blue dashed line. In the presence of the intermediate state (pre-equilibrium), the reaction occurs in two steps characterized by corresponding activation energies $E_{\mathrm{A} 1}, E_{\mathrm{A} 2}$ and energy releases $E_{1}, E_{2}$.

In case of a double-step reaction, one can assume that the times of activation of the first and of the second steps are sufficiently long for the system temperature to approach corresponding saturation values $\left(T_{1}\right.$ and $\left.T_{2}\right)$. In such a case, one can consider activation as a stepwise exothermic process with two consequent steps

$$
T_{0} \rightarrow T_{1} \rightarrow T_{2}
$$

with the solution for temperature in this case is Eq. (4). Note, the red line in Fig. 4(b) appear to be a smooth version of the stepwise heat source described by Eq. (3).

In the case of experiments with complex (dusty) plasmas, the first step is related to activation of the defect, while the second one is due to the flame front approach. The heat rate $Q(T)$ is determined by interplay between energy release (due to the nonreciprocity of effective interactions mediated by plasma flow) and dissipation (due to friction with neutral gas and heat conductivity in

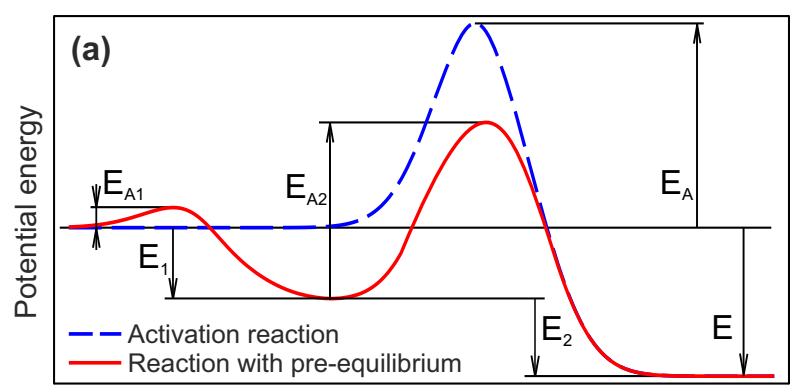

Reaction coordinate

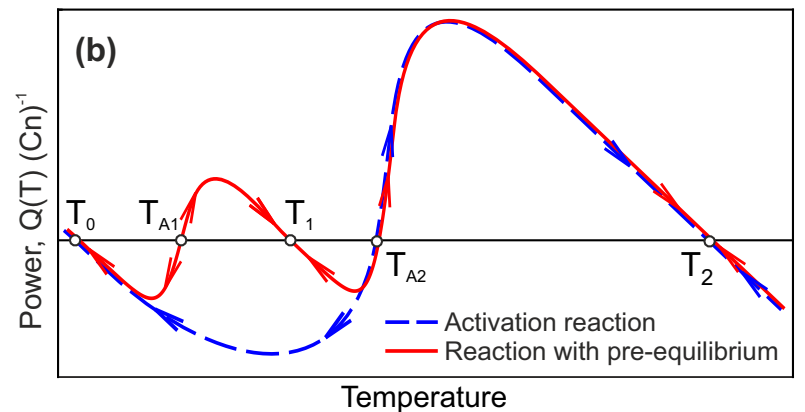

FIG. 4. Schematic diagram corresponding to the discussion about double-step exothermic activation (with pre-equilibrium): (a) scheme of pre-equilibrium state [27, (b) schematically shown power of heat source on temperature. Blue dashed and red solid lines correspond to the simple (one barrier) activation and double-step (activation with pre-equilibrium) case, respectively.

plasma crystal) 23, 25, 32. Of course, the complex (dusty) plasmas cannot provide a direct physical mapping taking into account all details of chemically-reactive systems (e.g., mass-altering concentrations of reacting chemicals), but, owing to the qualitatively adequate form of $Q(T)$ illustrated in Fig. 4, a similar thermal activation behavior and various thermal instabilities could be observed.

In analysis of our experimental and MD results, we used Eq. (3) with $T_{1}=T_{0}$ to fit the temperature profile in a solid without a thermal defect to obtain the parameters of profile corresponding to the flame front 23. Then, the double-step thermal behavior in vicinity of the defect was fitted using Eq. (4) with known parameters of the flame front (see Appendix D for details).

Examining the results in Figs. 2 and 3 , we clearly see the double-step thermal activation, which can be accurately described by Eq. (4). Although two steps in Eq. (4) are equal functionally, they are related to different processes in our experiment. At the first step, an acoustic compression wave induces an enhanced energy release from the defect, the local temperature in vicinity of the defect attains a Gaussian-like form (see solid orange lines in Figs. 2(b) and 3(b)) with the magnitude greater than its critical value (threshold of thermal explosion [25, 38]). The pre-equilibrium in the case of complex plasmas means that the intermediate state exists for a sufficiently long time before the system evolves to 

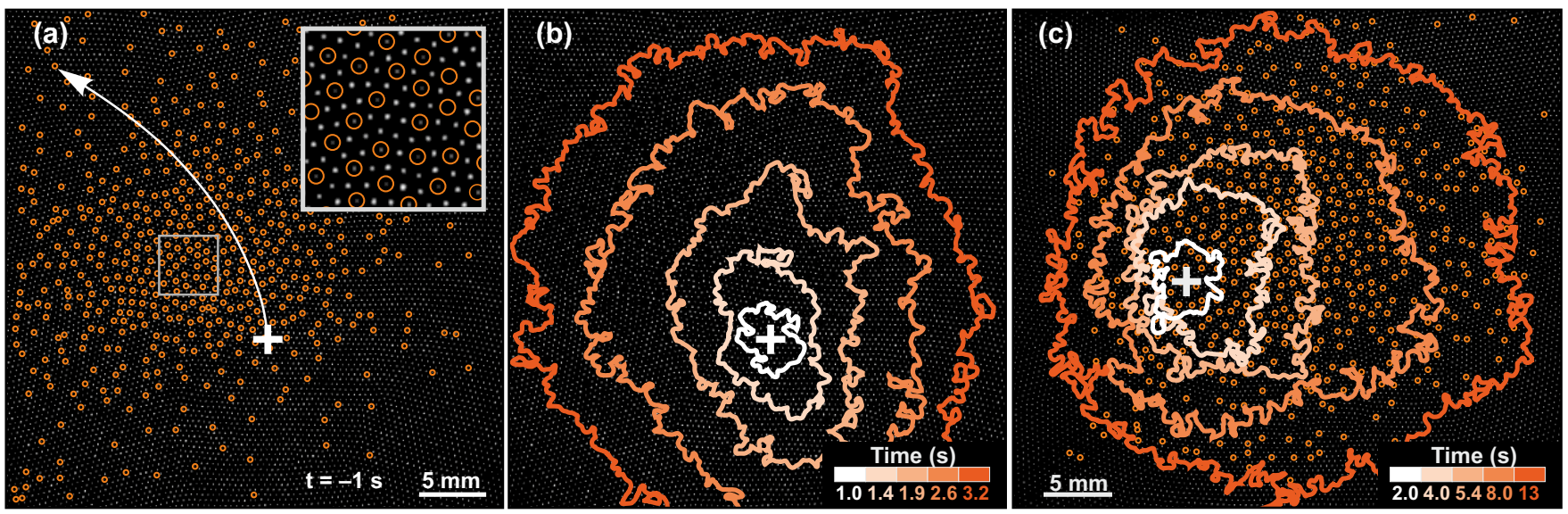

FIG. 5. Experiments with flames governed by defects: (a) Snapshot of crystal with spatially-inhomogeneous distribution of defects in the crystal (orange symbols) before the ignition. The ignition area is marked by the white cross and the curved arrow shows the orientation of the anisotropy axis for the fronts shown in (b). The inset shows a zoom on the area in the grey frame to highlight the mix of normal and heavier particles (thermal defects) governing the flame front propagation. (b) Color-coded fronts corresponding to the average thermal energy of particles $T=20 \mathrm{eV}$ at different times. (c) Experiment with stopped flame fronts. Details of description is the same as in (a) and (b). See Movies S3 and S4 [27]

the final (completely melted) state after the flame front approach (whose profile is shown by solid blue line in Figs. 2(b) and 3(b)). Since the near-threshold states can exist for a long time [25, the activated state can be accurately described by Eq. (4) with some effective parameters [23].

\section{FLAMES GOVERNED BY DEFECTS}

The results observed so far suggest that the defects have clear influence on the flame front propagation. This opens promising prospects for studies of flame fronts, whose direction and velocity are governed by the defects. To test this idea, we performed dedicated experiments with inhomogeneous defect distribution (see Appendix A for details).

Figure 5(a) presents the initial distribution of the defects (orange circles), where the region of ignition, and direction of the flame front propagation are shown by the white cross and the arrow, respectively. The isotherms (at the temperature of $T=20 \mathrm{eV}$ ), shown in Fig. 5(b) at different times, demonstrate that the flame front is deformed along the defect cloud. The influence of the defects can be seen even more clearly in Fig. 5.(c) presenting results of experiment with "stopped" flame front. Here, the front propagated to the center of the defect cloud and then was halted, since the crystal without the defects was stable under the conditions of this experiment. This result proves that the defects do indeed reduce the activation threshold. Since each defect reduces thermal activation threshold in its vicinity, their inhomogeneous distribution modulates spatially the field of thermal threshold and, thus, local velocity of the front propagation. As in the previous experiments, contours of the steady state (formed at a sufficiently long time) are determined by the balance of the energy release (due to nonreciprocity) and dissipation (due to Epstein damping). Therefore, by changing spatial distribution of the defects, one can govern direction and the form of the flame front as well as vary its averaged (effective) propagation velocity.

\section{THE ROLES OF PLASMA WAKES AND DEFECT CONCENTRATION}

The observed thermal activation occurs near a thermal defect in the complex plasma due to the enhanced particle-wake interactions illustrated in Fig. 1. An analogous effect in reactive solids might be associated with a locally-enhanced chemical activity (e.g., due to reduced energy barriers in the presence of intermediates, as shown in Fig. 4 leading to more intensive chemical reactions and, thus, to a locally-enhanced heating. In the complex (dusty) plasmas, such behavior corresponds to a some range of particle charge, plasma wakes, and vertical confinement parameters. Consequently, we performed additional MD simulations to reveal the role of the wake parameters and the defect concentration on the thermal dynamics and on the velocity of non-equilibrium melting front propagation (flame front velocity).

Specifically, we focused on a role of the dimensionless charge of the wakes $q_{D}$ (see Appendix B for details), since, comparing to the wake length $h_{D}, q_{D}$-value most strongly affects the energy release due to the nonreciprocity of interactions. The effect of $q_{D}$-value on the evolution of the thermal energy in the vicinity of the defect obtained from the MD simulations for the systems with the same initial conditions is shown in Fig. 6. Blue and red symbols correspond to the crystalline area and thermal 


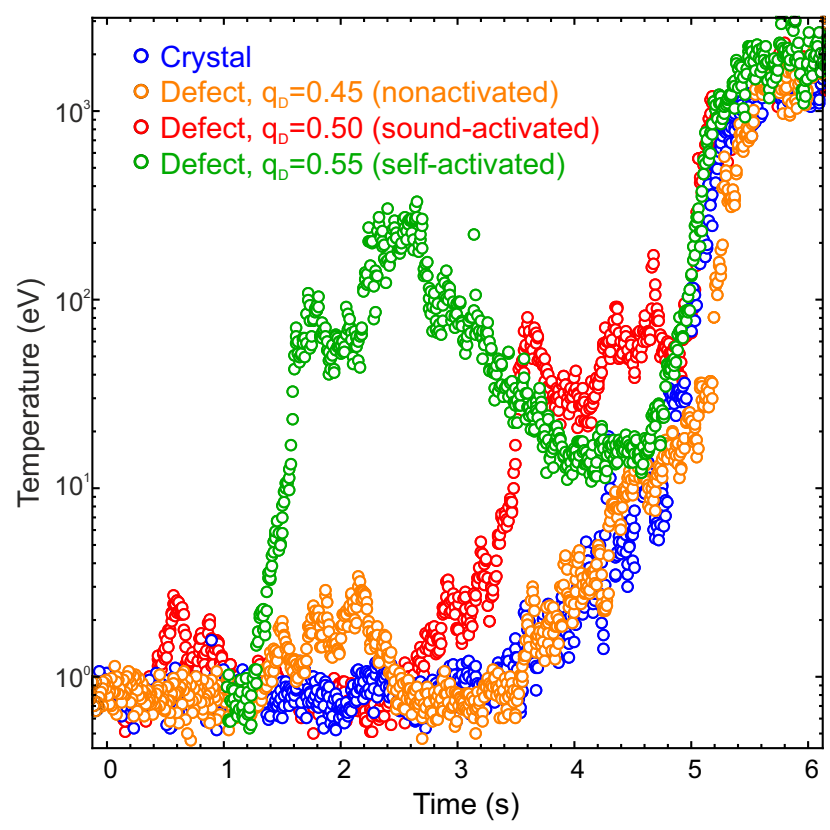

FIG. 6. Thermal energy evolution in the vicinity of the defect with different charge $q_{D}$ : The blue and red symbols reproduce the points shown in Fig. 3(a) for the crystal (without defects) and the sound-activated defect $\left(q_{D}=0.5\right)$. Results of MD simulation for $q_{D}=0.45$ (the non-activated defect) and 0.55 (the self-activated defect) are shown by the orange and green symbols. Simulations were performed for the same initial states, but with the different $q_{D}$ values.

defect with $q_{D}=0.5$ (the same as shown in Fig. 3(a), reproduced here for comparison with other $q_{D}$-values). The results of simulations with $q_{D}=0.45$ and 0.55 are shown by the orange and green symbols, respectively.

Based on the results of our simulations, one may conclude that, depending on $q_{D}$, the thermal defects can exhibit different dynamics, as illustrated in Fig. 6. At low values of $q_{D} \lesssim 0.45$, the energy release is insufficient to put the defect in the pre-activated state even in the presence of the sound generated due to the thermoacoustic instability during propagation of the nonequilibrium melting 26]. The typical thermal behavior in this case (orange symbols) is non-activated and practically the same as that in the crystal (blue symbols), as seen in Fig. 6. Since the plasma wakes are weak, the defects behave like normal particles, but the thermal energy in their vicinity is slightly elevated and exhibits weak (lowfrequency) spontaneous fluctuations.

In the narrow range of $q_{D}$-values $\left(0.45 \lesssim q_{D} \lesssim 0.5\right.$ in our case), the energy release rate becomes sufficiently large and the defect can be activated (being put in the pre-activated state, existing for a sufficiently long time, as has been observed in our experiments and simulations and illustrated in Figs. 1. 3 by the sound. The typical example of such sound-activated thermal behavior is shown by the red symbols in Fig. 6. In this case, the sound wave is necessary for the activation of defect; otherwise

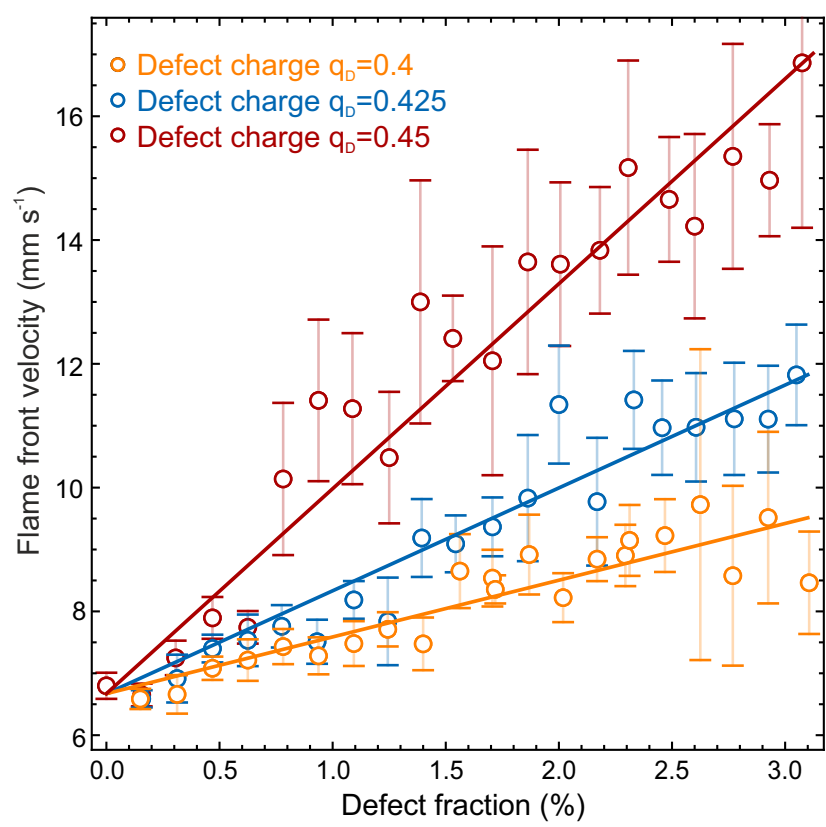

FIG. 7. The effect of the defect concentration on the velocity of the nonequilibrium melting (flame front) propagation: The orange, blue, and red symbols demonstrate the MD results for the velocity of the flame front propagation as a function of defect concentration with $q_{D}=0.4$, 0.425 , and 0.45 , respectively. The lines are the corresponding linear fits.

it can remain non-activated for a long time. For instance, in the case of our simulations, some defects could exist non-activated the entire simulation time, but be easily activated by a sound wave.

Finally, starting from a certain $q_{D}\left(q_{D} \gtrsim 0.5\right.$ in our simulations), the energy release due to nonreciprocal interactions gets so strong that the defect becomes selfactivated. Such defect cannot exist in a non-activated state and exhibits a self-propelled motion, like an active particle (the mechanism providing this activity has been recently reported in Ref. [39]). This case, shown by the green symbols in Fig. 6, typically exhibits a developed activity of the particle immediately since the simulation onset.

The concentration of thermal defects is also an important factor determining collective thermal behavior, as we observed experimentally and illustrated in Fig. 5 . Using MD simulations, we analyzed the velocity of the flame front propagation on the defect concentration at different charges of the defect wakes, $q_{D}=0.4,0.425$, and 0.45 , corresponding to the defects being sound-activated. The results for the flame front velocity vs the defect concentration are shown in Fig. 7, where the symbols are the MD results and the lines are linear fits. One can see that, the flame front velocity $v_{\text {fr }}$ exhibits linear increase with growth in $q_{D}$ and is commensurate with increase of flame front velocity with the energy release, similar to chemically reactive matter $[23,38$. 
Thus, to conclude, parameters of the plasma wakes can switch the defect dynamics between three regimes: (i) non-activated, (ii) sound-activated, and (iii) selfactivated. In the case of sound-activated defects, at some concentration of the defects, the regime of flame front propagation can be changed, since the defects constantly become activated by the sound ahead of the melting front, thus, increasing the energy release and the flame front velocity. The double-step thermal behavior turned out to exist between the non-activated and self-propelled regimes of individual particle dynamics. This complex dynamics is accompanied by the interplay between the dissipative and thermodynamic phase transitions (the thermal activation and the melting of the crystal), and we leave a more detailed study of transitions between the discussed regimes for future work.

\section{CONCLUSIONS}

Here we revealed a remarkable analogy between combined instabilities in complex (dusty) plasmas and chemical reactions with pre-equilibrium. For a long time, instabilities in such systems, have been considered as parasitic effects in particle-resolved studies and a number of phenomena (e.g. crystalline and fluid MCI, thermoacoustic instability, and thermal explosion) had to be studied separately. We recently demonstrated [23, 24, 26] that such instabilities can serve as model systems to study thermoacoustic behaviour in chemically-reactive media and flame fronts. Here, we show for the first time that combined instabilities (crystalline MCI - fluid MCI - thermoacoustic instability - thermal explosion) create conditions for thermal activation of heavier particles, which can exhibit behaviour of thermal defects, providing physical analogy with double-step exothermic chemical reactions (reactions with pre-equilibrium).

Although we studied the activation processes and the cascades of instabilities in monolayer complex plasmas, a qualitatively similar behavior should be expected in $3 \mathrm{D}$ systems. This is because the observed activation of the defects is based on a few generic phenomena, intrinsic for both 2D and 3D systems, including the threshold thermal activation (in the plasma, it is realized with the crystalline and fluid MCI [34, 35]), the flame front formation (the nonequilibrium melting front formation in the plasma [23]), generation of sound waves due to thermoacoustic instability (it is also a generic phenomenon for the complex plasmas and chemically-reactive media, as was shown in Ref. [26]), and activation of the thermal defects (in the case of the complex plasmas, thermal-explosionlike behavior was reported in Ref. [25]).

We demonstrated that thermal defects change kinetics of energy release in exothermic reactions, thus, affecting flame front velocity and structure. The results open exciting prospects to study complex combustion processes (e.g., combination of thermal activation with melting and chemical reactions), role of thermal defects in propaga- tion of intensive exothermic reactions and acceleration of flame fronts in energy materials on particle-resolved (microscopic) level. Crucially, our findings demonstrate that operating complex (dusty) plasmas in such a regime not only provides a platform for model experiments, but can suggest new directions for future experiments with chemically-reactive media.

It is important to point out, that activation behavior is intrinsic for nonreciprocally-interacting systems, broadly presented in nature and technical systems, from complex plasmas and active matter to multi-agent systems. Therefore, present results are of high relevance beyond the immediate area of present study. We hope that our work will stimulate theoretical and experimental studies in related areas if physics of plasmas, physical chemistry, chemical physics, soft matter, and materials science.

\section{ACKNOWLEDGMENTS}

Study was supported by Russian Science Foundation Grant No. 17-19-01691. S.O.Y is grateful to Acad. V. Brazhkin for fruitful discussions.

\section{AUTHOR CONTRIBUTIONS}

E.V.Y. performed experiments; N.P.K. and K.P. performed MD simulations; N.P.K., K.P., P.V.O., and S.O.Y. processed experimental results and MD simulations; E.V.Y., N.P.K., A.V.S., and S.O.Y. analyzed and discussed the results; S.O.Y. conceived, directed the research, developed theory, and wrote the manuscript. All authors reviewed the manuscript.

\section{Appendix A: Experimental details}

We used a modified GEC chamber filled with argon gas, in a capacitively coupled RF glow discharge at 13.56 $\mathrm{MHz}$. The top electrode was a grounded ring and the bottom (powered) electrode was an aluminium disk with a diameter of $\simeq 220 \mathrm{~mm}$. The argon gas pressure and the forward rf power were in the range of $0.5-2.5 \mathrm{~Pa}$ and $5-25 \mathrm{~W}$, respectively. More detailed description of the setup and similar experiments are reported in Refs. 23. 26, 34, 40.

Thermal activation of a defect.- Melamineformaldehyde spherical particles of the diameter $9.19 \pm 0.14 \mu \mathrm{m}$ and mass $m=6.1 \times 10^{-10} \mathrm{~g}$ were injected in the discharge. Due to the large negative charges acquired in the plasma discharge, the particles were confined in the sheath above the rf electrode. The microparticles in the layer were illuminated using a horizontal laser sheet and imaged by a Photron FASTCAM SA6 camera (through a window at the top of the chamber) at 250 frames per second. We used an additional side-view camera Edmund Optics 0413M to 
verify that the particles formed a single layer. After heavy agglomerates of the particles were removed, $2 \mathrm{D}$ plasma crystal with diameter about $100 \mathrm{~mm}$ was formed. In total, we performed five experiments providing qualitatively the same results. Details of similar experiments were reported in Refs. 34, 40, 41, and in our recent papers [23, 26].

In the experiment with a heavier particle (Figs. 1 and 2. Movie S1 27]) acting as a defect, the crystalline modecoupling instability (MCI), followed by propagation of the "flame front" in complex (dusty) plasma crystal, was observed at a pressure 1.1 $\mathrm{Pa}$ and forward rf power of $22 \mathrm{~W}$. Crystalline MCI was triggered in the densest (central) region of the crystal and then was expanding outward. Average particle distance in the crystal was $a \simeq 410 \pm 25 \mu \mathrm{m}$, number of initially tracked particles was $N=13880$.

We employed the standard approach 42, 43 to obtain the particle coordinates and velocities in each frame. Thermal and hydrodynamic energies of particles, $T$ and $K$, were found using the relationships:

$$
T=\frac{m}{2}\left\langle\left(\mathbf{v}_{i}-\left\langle\mathbf{v}_{i}\right\rangle\right)^{2}\right\rangle, \quad K=\frac{m}{2}\left\langle\mathbf{v}_{i}\right\rangle^{2},
$$

where $\mathbf{v}_{i}$ is the instantaneous in-plane velocity of the $i$ th particle, and $\left\langle\mathbf{v}_{i}\right\rangle$ is the instantaneous average ("local hydrodynamic") velocity of all particles within radius of $2.4 a$ around the $i$ th particle. Density was calculated in the same manner.

Determination of particle charge and screening parameter.- Particle charge number $Z$ and the screening parameter $\kappa=a / \lambda_{\mathrm{D}}$ (where $a$ is the interparticle distance and $\lambda_{\mathrm{D}}$ the Debye screening length) were deduced from the fluctuation spectra using the standard approach described in Ref. 41, 44]

Flames governed by defects. - Experiment with directed flame front (shown in Figs. 5(a) and 5(b) of the main text and in Movie S3 27]) was performed at forward power of $20 \mathrm{~W}$ and the gas pressure of $1.0 \mathrm{~Pa}$. Following purification by the standard method (i.e. reducing discharge power to drop agglomerates of microparticles) only monodisperse particles and two-particle agglomerates were left. Agglomerates then played role of thermal defects, which were randomly and inhomogeneously distributed after crystallization in thus assembled crystal (see Movie S3 [27]).

In the experiment with the "stopped" flame front (Fig. 5(c) and Movie S4 [27]), crystalline MCI started at forward power of $15 \mathrm{~W}$ and argon pressure of $1.6 \mathrm{~Pa}$. Preparation of the crystal for this experiment was done in the same manner as that in experiment with the directed flame front.

\section{Appendix B: Details of MD simulations}

Molecular dynamics (MD) simulations were performed using the following interparticle potential [23, 26]:

$$
\varphi(\mathbf{r})=\epsilon\left[\frac{e^{-r / \lambda_{D}}}{r / \lambda_{D}}-q \frac{e^{-r_{w} / \lambda_{D}}}{r_{w} / \lambda_{D}}\left(1+b \frac{e^{-r_{w} / \lambda_{D}}}{r_{w} / \lambda_{D}}\right)^{-1}\right],
$$

where $\mathbf{r}$ is the vector between two particles, $\lambda_{D}=300 \mu \mathrm{m}$ is the Debye screening length, $\epsilon=e^{2} Z^{2} / 4 \pi \varepsilon_{0} \lambda_{D}=$ $1950 \mathrm{eV}$ is the energy scale of the interaction, $\varepsilon_{0}$ is the electric constant, $Z=2 \times 10^{4}$ is the particle charge number, $q=0.36$ is the relative charge of plasma wake, $r_{w}=\left|\mathbf{r}-h \mathbf{e}_{z}\right|$ is the distance to the wake of a neighbor particle, $h=106 \mu \mathrm{m}$ is the effective wake length and $b=0.05$ is cut-off parameter of the wake model.

The scheme of forces acting between the couple of charged dusty particles in plasma flow is illustrated in Figure 8. Plasma wakes, formed downstream the particles in the plasma flow, play a role of the third body and affect the interparticle interactions, making them nonreciprocal. This means that the action and reaction for the effective forces are violated, the system is thus non-conservative, and energy can transfer effectively from the plasma stream into the kinetic energy of moving microparticles.

In reality, the interaction between particles in moving plasma is more complicated than a combination of two spatially-shifted Yukawa potentials (even with account of truncation), since it strongly depends on the plasma parameters, as was reported in Ref. 31. However, Eq. (C1) sufficiently accurately describes formation and propagation of flame fronts in crystals 23, activation thermal behavior of fluid complex (dusty) plasmas [26], and allows to simulate a broad range of activation phenomena

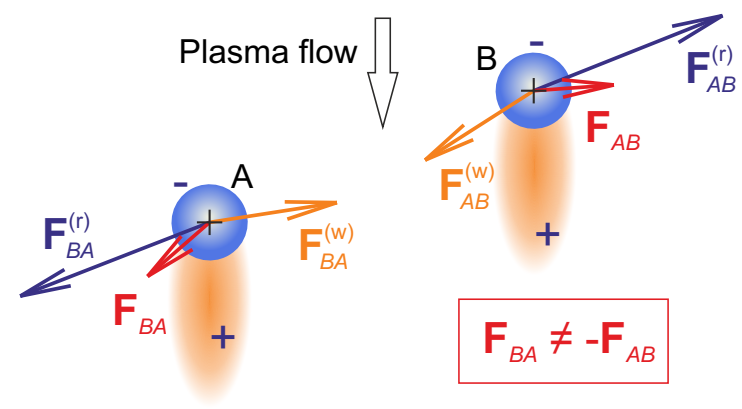

FIG. 8. Schematic diagram of forces acting between two particles in plasma flow: Two negatively charged particles (colored in blue) in plasma flow interact nonreciprocally due to the presence of positively charged wakes (shown by orange clouds). $\mathbf{F}^{(r)}$ (with corresponding indices) are the reciprocal (repulsive) forces acting between the particles A and $\mathrm{B}, \mathbf{F}^{(w)}$ denote (attractive) interactions between (negativelycharged) particles and (positively-charged) wakes. One can see that particle-wake interactions affect the reciprocity of action and reaction for effective forces, $\mathbf{F}_{A B} \neq-\mathbf{F}_{B A}$. 
caused by particle-wake interactions.

To reproduce formation of a horizontal layer corresponding to our experiment, the particles were placed into the potential well

$$
U(x, y, z)=\frac{1}{2} m \Omega^{2}\left[z^{2}+\alpha\left(x^{2}+y^{2}\right)\right],
$$

where $\Omega=36.8 \mathrm{~Hz}$ is the frequency of the vertical oscillations in the limit $k \rightarrow 0$, which can be obtained from the experimental fluctuation spectra, $\alpha=2.3 \times 10^{-5}$ is the constant introduced to account for a weak horizontal confinement and $m=6.1 \times 10^{-10} \mathrm{~g}$ is particles masses.

The simulations were performed for $N=4 \times 10^{4}$ particles in the $N V T$ ensemble in the Langevin thermostat with the equilibrium temperature $T_{0}=0.8 \mathrm{meV}$. Experimentally-relevant value of the Epstein damping rate $\gamma_{d}=1.2 \mathrm{~s}^{-1}$ was used in our simulations. A numerical time step was $\Delta t=13 \mu \mathrm{s}$.

We introduced one larger particle (defect, D) in our system in order to simulate thermal activation of defects formed by heavier particles. We assumed that the diameter of the D-particle is approximately 1.3 times larger than that of normal particles, since the diameter of a particle twice the mass of a normal particle is $2^{1 / 3} \simeq 1.3$ times larger than that of individual particles, while dust charge and corresponding Epstein rate are proportional to the diameter and its square [6. Hence, mass, particle charge number, and damping rate for D-particle can be taken as $m_{\mathrm{D}}=2 m, Z_{\mathrm{D}}=1.3 Z$, and $\gamma_{d, \mathrm{D}}=1.3^{2} \gamma_{d}$. The frequency in the potential well $(\mathrm{B} 2)$ for D-particle was set as $\Omega_{\mathrm{D}}=\sqrt{1.3 / 2} \Omega$ and we added an additional force $\mathbf{f}_{\mathrm{add}, \mathrm{D}}=-m g \mathbf{e}_{z}$ acting on D-particle, where $g$ is the gravitational acceleration. Parameters of interaction potential for the case of the defect and normal particle were chosen as $h_{\mathrm{D}}=h, q_{\mathrm{D}}=0.5, \epsilon_{\mathrm{D}}=1.3 \epsilon$.

The initial state of the system was formed as a monolayer in $z=0$ plane. The first $1.5 \times 10^{6}$ time steps were performed at large values of the damping rate $\gamma_{R}=6 \gamma_{d}$ for all particles and were used to establish the Boltzmann distribution in the potential well. After this, the following $5 \times 10^{5}$ time steps with damping rates $\gamma_{d}$ and $\gamma_{d, \mathrm{D}}$ for normal and D-particles, respectively, were used to obtain MD data for analysis (See Movie S2 [27]).

To identify the influence of the defect concentration on the velocity of flame front propagation, the MD simulations were performed in a system without confinement in the horizontal plane ( $\alpha=0$ in Eq. (B2 $)$ in the simulation box with periodic boundary conditions. At the initial step, the particles were placed in the horizontal plane $z=0$ and arranged into an ideal hexagonal lattice (lattice constant was equal to $387 \mu \mathrm{m}$ ) occupying the whole system in the $(x, y)$-plane. The ratio of simulation box edges $L_{x}$ to $L_{y}$ was equal to 11.5 ; some of the particles were turned into defects. The parameters for the defectdefect interaction were set as $\epsilon_{\mathrm{DD}}=1.3^{2} \epsilon, q_{\mathrm{DD}}=q_{\mathrm{D}}$ and $h_{\mathrm{DD}}=h$. To start with, $2 \times 10^{4}$ simulation steps with increased Epstein rate (up to $50 \gamma_{d}$ ) were performed to equilibrate the system. After that, the Epstein rate was returned to (the experimentally-relevant) $\gamma_{d}$-value and velocities of the particles located in the central region of the system were increased to "ignite" the system. As a result, the propagating flame front was formed in the system and its average velocity could have been measured in the same manner as in Refs. [10, 23].

\section{Appendix C: Solution of Eq. (1) for double-step thermal activation}

Consider solution of the problem for the one-step case, illustrated in Fig. 4 Equation (1) can be written in the following form:

$$
\frac{\partial T}{\partial t}=\frac{Q(T)}{C n}+\chi \frac{\partial^{2} T}{\partial r^{2}} .
$$

In case of the one-step exothermic chemical reaction (flame front), the heat source can be used in the steplike form, while the dissipation (from chemically-reactive media to the environment) grows linearly with temperature:

$$
\frac{Q(T)}{C n}= \begin{cases}-\alpha\left(T-T_{0}\right), & T<T_{A} ; \\ q-\alpha\left(T-T_{0}\right), & T>T_{A},\end{cases}
$$

where $T_{A}$ is the activation temperature, $\alpha$ is a dissipation coefficient, and the heat source can be represented in the form $q=\alpha\left(T_{1}-T_{0}\right)$.

Using continuity of the self-similar temperature profile $T\left(t-r / v_{\text {fr }}\right) \equiv T(\tau)$ (here $v_{\text {fr }}$ is the front velocity) and its derivative at the activation point $\left(T=T_{A}\right.$ at $\left.\tau=0\right)$, one can obtain the following solution of Eq. (C1):

$$
T(\tau)= \begin{cases}T_{0}+\frac{T_{1}-T_{0}}{1+\lambda_{1} / \lambda_{2}} e^{\lambda_{1} \tau}, & \tau<0 \\ T_{1}-\frac{\lambda_{1}}{\lambda_{2}} \frac{T_{1}-T_{0}}{1+\lambda_{1} / \lambda_{2}} e^{-\lambda_{2} \tau}, & \tau>0\end{cases}
$$

where

$$
\lambda_{1,2}=\left(\sqrt{1+4 \alpha \chi / v_{\mathrm{fr}}^{2}} \pm 1\right) v_{\mathrm{fr}}^{2} / 2 \chi
$$

and $T_{A}-T_{0}=\left(T_{1}-T_{0}\right) /\left(1+\lambda_{1} / \lambda_{2}\right)$.

In case of temperature observation at a fixed point, we obtain the following time-dependence at the point (with a fixed $r$ )

$$
T(t)= \begin{cases}T_{0}+\frac{T_{1}-T_{0}}{1+\lambda_{1} / \lambda_{2}} e^{\lambda_{1}\left(t-t_{A}\right)}, & t<t_{A} ; \\ T_{1}-\frac{\lambda_{1}}{\lambda_{2}} \frac{T_{1}-T_{0}}{1+\lambda_{1} / \lambda_{2}} e^{-\lambda_{2}\left(t-t_{A}\right)}, & t>t_{A},\end{cases}
$$

where $t_{A}$ is the time of flame front approach.

\section{Appendix D: Details of fitting procedure}

Experimental data for temperature evolution in the areas of interests were analyzed using Eq. (4). At the 
TABLE I. Parameters if fits obtained from experimental and MD results

\begin{tabular}{c|c|c|c|c|c|c|c|c|c|c|c}
\hline Region of analysis & $T_{0}, \mathrm{eV}$ & $T_{1}, \mathrm{eV}$ & $T_{2}, \mathrm{eV}$ & $\lambda_{3}, \mathrm{~s}^{-1}$ & $\lambda_{4}, \mathrm{~s}^{-1}$ & $\lambda_{1}, \mathrm{~s}^{-1}$ & $\lambda_{2}, \mathrm{~s}^{-1}$ & $t_{1}, \mathrm{~s}$ & $t_{2}, \mathrm{~s}$ & $T_{A 1}, \mathrm{eV}$ & $T_{A 2}, \mathrm{eV}$ \\
\hline \hline Area A (crystal), Exp. & 0.3 & - & 555 & 3.81 & 10 & - & - & - & 6.77 & - & 400 \\
Area B (defects), Exp. & 0.3 & 21 & 555 & 3.81 & 10 & 23.56 & 5 & 5.22 & 6.57 & 3.93 & 400 \\
\hline \hline Area A (crystal), MD & 0.87 & - & 1660 & 4.86 & 10 & - & - & - & 5.6 & - & 1116 \\
Area B (defects), MD & 0.87 & 40.2 & 1660 & 4.86 & 10 & 5.86 & 5 & 3.53 & 5.44 & 18.98 & 1116 \\
\hline \hline
\end{tabular}

first step, we analyzed the crystalline areas (in this case, $T_{1}=T_{0}$ in Eq. (4)). The temperatures $T_{0}$ and $T_{2}$ were obtained by averaging the temperature points before the activation and behind the flame front. Then, the coefficients $\lambda_{3,4}$ were obtained by the least square method (with the error bars calculated for the energy in the region of interest).

After this, the obtained (crystalline) parameters $\lambda_{3,4}$ and $T_{2}$ were used for determination of the coefficients $\lambda_{1,2}$ and of $T_{1}$. The temperature $T_{0}$ for areas with defects was calculated in the same manner as for crystalline areas.

The results of our MD simulations were analyzed in the same manner as experimental ones and obtained parameters are presented in Table I. MD simulations yield an expectedly enhanced energy release, because of specificity of the potential (B1).
[1] J. Agarwal, J. M. Turney, and H. F. Schaefer, The Journal of Physical Chemistry Letters 2, 2587 (2011), https://doi.org/10.1021/jz201124j.

[2] M. M. Kuklja and S. N. Rashkeev, The Journal of Physical Chemistry Letters 1, 363 (2010).

[3] M. B. Toftegaard, J. Brix, P. A. Jensen, P. Glarborg, and A. D. Jensen, Progress in Energy and Combustion Science 36, 581 (2010)

[4] G. E. Morfill and A. V. Ivlev, Rev. Mod. Phys. 81, 1353 (2009)

[5] M. Chaudhuri, A. V. Ivlev, S. A. Khrapak, H. M. Thomas, and G. E. Morfill, Soft Matter 7, 1287 (2011)

[6] A. Ivlev, H. Löwen, G. Morfill, and C. P. Royall, Complex plasmas and Colloidal dispersions: particle-resolved studies of classical liquids and solids (Series in soft condensed matter) (Singapore: Word Scientific, 2012).

[7] A. Ivlev, V. Nosenko, and T. Rocker, Contributions to Plasma Physics 55, 35 (2015)

[8] V. A. Schweigert, I. V. Schweigert, A. Melzer, A. Homann, and A. Piel, Phys. Rev. Lett. 80, 5345 (1998)

[9] V. Nosenko, S. K. Zhdanov, A. V. Ivlev, C. A. Knapek, and G. E. Morfill, Phys. Rev. Lett. 103, 015001 (2009).

[10] J. D. Williams, E. Thomas, L. Couëdel, A. V. Ivlev, S. K. Zhdanov, V. Nosenko, H. M. Thomas, and G. E. Morfill, Phys. Rev. E 86, 046401 (2012)

[11] A. Melzer, A. Homann, and A. Piel, Phys. Rev. E 53, 2757 (1996)

[12] P. Hartmann, A. Douglass, J. C. Reyes, L. S. Matthews, T. W. Hyde, A. Kovács, and Z. Donkó, Phys. Rev. Lett. 105, 115004 (2010)

[13] M. Rubin-Zuzic, G. E. Morfill, A. V. Ivlev, R. Pompl, B. A. Klumov, W. Bunk, H. M. Thomas, H. Rothermel, O. Havnes, and A. Fouquét, Nature Physics 2, 181 (2006)

[14] S. Nunomura, D. Samsonov, S. Zhdanov, and G. Morfill, Phys. Rev. Lett. 95, 025003 (2005).

[15] V. Nosenko, S. Zhdanov, A. V. Ivlev, G. Morfill, J. Goree, and A. Piel, Phys. Rev. Lett. 100, 025003 (2008).

[16] V. Nosenko, S. Zhdanov, and G. Morfill, Phys. Rev. Lett. 99, $025002(2007)$.
[17] P. Hartmann, A. Z. Kovács, A. M. Douglass, J. C. Reyes, L. S. Matthews, and T. W. Hyde, Phys. Rev. Lett. 113, 025002 (2014)

[18] Y.-F. Lin, A. Ivlev, H. Löwen, L. Hong, and C.-R. Du, EPL (Europhysics Letters) 123, 35001 (2018).

[19] H. Huang, A. V. Ivlev, V. Nosenko, Y.-F. Lin, and C.-R. Du, Physics of Plasmas 26, 013702 (2019)

[20] A. Wysocki, C. Räth, A. V. Ivlev, K. R. Sütterlin, H. M. Thomas, S. Khrapak, S. Zhdanov, V. E. Fortov, A. M. Lipaev, V. I. Molotkov, O. F. Petrov, H. Löwen, and G. E. Morfill, Phys. Rev. Lett. 105, 045001 (2010).

[21] A. V. Ivlev, S. K. Zhdanov, H. M. Thomas, and G. E. Morfill, EPL (Europhysics Letters) 85, 45001 (2009).

[22] C. Killer, T. Bockwoldt, S. Schütt, M. Himpel, A. Melzer, and A. Piel, Physical Review Letters 116, 115002 (2016)

[23] S. O. Yurchenko, E. V. Yakovlev, L. Couëdel, N. P. Kryuchkov, A. M. Lipaev, V. N. Naumkin, A. Y. Kislov, P. V. Ovcharov, K. I. Zaytsev, E. V. Vorob'ev, G. E. Morfill, and A. V. Ivlev, Phys. Rev. E 96, 043201 (2017).

[24] L. Couedel, V. M. Nosenko, S. Zhdanov, A. V. Ivlev, I. Laut, E. V. Yakovlev, N. P. Kryuchkov, P. V. Ovcharov, A. M. Lipaev, and S. O. Yurchenko, PhysicsUspekhi (2019), 10.3367/ufne.2019.01.038520.

[25] L. Couëdel, V. Nosenko, M. Rubin-Zuzic, S. Zhdanov, Y. Elskens, T. Hall, and A. V. Ivlev, Phys. Rev. E 97, 043206 (2018).

[26] N. P. Kryuchkov, E. V. Yakovlev, E. A. Gorbunov, L. Couëdel, A. M. Lipaev, and S. O. Yurchenko, Phys. Rev. Lett. 121, 075003 (2018)

[27] See Supplemental Material at (URL) for Movies S1-S4.

[28] M. Lampe, G. Joyce, G. Ganguli, and V. Gavrishchaka, Physics of Plasmas 7, 3851 (2000).

[29] G. A. Hebner, M. E. Riley, and B. M. Marder, Phys. Rev. E 68, 016403 (2003).

[30] A. V. Ivlev, J. Bartnick, M. Heinen, C.-R. Du, V. Nosenko, and H. Löwen, Phys. Rev. X 5, 011035 (2015)

[31] R. Kompaneets, G. E. Morfill, and A. V. Ivlev, Phys. Rev. E 93, 063201 (2016).

[32] N. P. Kryuchkov, A. V. Ivlev, and S. O. Yurchenko, Soft Matter 14, 9720 (2018) 
[33] A. V. Ivlev and G. Morfill, Phys. Rev. E 63, 016409 (2000)

[34] L. Couëdel, V. Nosenko, A. V. Ivlev, S. K. Zhdanov, H. M. Thomas, and G. E. Morfill, Phys. Rev. Lett. 104, $195001(2010)$

[35] A. V. Ivlev, S. K. Zhdanov, M. Lampe, and G. E. Morfill, Phys. Rev. Lett. 113, 135002 (2014).

[36] P. Atkins, J. d. Paula, and J. Keeler, Atkins' Physical Chemistry (11th Edition) (University Press, Oxford, 2017).

[37] L. D. Landau and E. M. Lifshitz, Fluid Mechanics. Vol. 6 (2nd ed.) (Elsevier, Oxford, 1987).

[38] A. V. Ivlev, T. B. Röcker, A. Vasyunin, and P. Caselli, The Astrophysical Journal 805, 59 (2015).

[39] I. Laut, C. Räth, S. K. Zhdanov, V. Nosenko, G. E. Morfill, and H. M. Thomas, Phys. Rev. Lett. 118, 075002
$(2017)$

[40] T. B. Röcker, L. Couëdel, S. K. Zhdanov, V. Nosenko, A. V. Ivlev, H. M. Thomas, and G. E. Morfill, EPL (Europhysics Letters) 106, 45001 (2014)

[41] L. Couëdel, S. K. Zhdanov, A. V. Ivlev, V. Nosenko, H. M. Thomas, and G. E. Morfill, Physics of Plasmas 18, 083707 (2011), 10.1063/1.3620406

[42] S. S. Rogers, T. A. Waigh, X. Zhao, and J. R. Lu, Physical Biology 4, 220 (2007)

[43] Y. Feng, J. Goree, and B. Liu, Review http://dx.doi.org/10.1063/1.2735920.

[44] S. K. Zhdanov, A. V. Ivlev, and G. E. Morfill, Physics of Plasmas 16, 083706 (2009), 10.1063/1.3205894. 